\title{
Towards Lagrangian Large Vortex Simulation
}

\author{
John R. Mansfield, Omar M. Knio and Charles Meneveau \\ Department of Mechanical Engineering \\ The Johns Hopkins University \\ Baltimore, MD 21218, USA
}

\begin{abstract}
Construction of a Lagrangian large-eddy-simulation scheme is performed based on filtering the vorticity transport equation and using simple eddy-diffusivity models to account for subgridscale "Helmholtz" stresses. The resulting governing equations are simulated using a threedimensional particle method which discretizes the vorticity field into Lagrangian vortex elements of finite overlapping cores. Vortex elements are transported along particle trajectories, while their vorticities evolve according to stretching and tilting by the resolved scales, molecular diffusion, and subgrid-scale stresses. The behavior of the model is discussed in light of limited three-dimensional computations of transitional vortex rings. It is shown that the model can capture the large-scale features of the flow without requiring an excessively large number of elements.
\end{abstract}

\section{Introduction}

Large-eddy-simulation (LES) generally aims at overcoming the scale disparity of turbulent flow by constructing models which describe the evolution of the larger scales of motion. Since the smallest scales of motion fall below the computational mesh size, the impact of unresolved or subgrid scales must be modeled explicitly. For the purpose of the present discussion, we shall consider simulation tools which are suitable for LES of time-dependent, three-dimensional incompressible flows.

The most common LES approach is based on spatially filtering the equations of motion $[13,16]$. Due to the nonlinearity of the momentum equations, a stress term appears in the filtered equations which includes a direct contribution from unresolved scales. This is the analogue of the well-known closure problem which affects the Reynolds-averaged equations of motion, and it is this unknown stress which must be modeled exclusively in terms of resolved, large-scale quantities. When the filtering operation commutes with spatial differentiation, the model consists of the original equations of motion with an added subgrid-scale stress term.

Most previous applications of LES have been performed using grid-based numerical techniques. On the other hand, particle-based, Lagrangian LES schemes remain scarce. The original efforts towards the development of vorticity-based, Lagrangian LES schemes are due to Chorin [3, 4]. These have led, in particular, to the well-known "hairpin-removal" schemes. The latter are motivated by renormalization of the vorticity evolution equations which, like spatial filtering, aims at "absorbing" the effect of the smallest lengthscales by constructing "effective" equations of motion. In its basic form, Chorin's hairpin removal scheme essentially consists of a filament-based simulation together with a local mesh redistribution algorithm. The redistribution algorithm acts on the geometry of the filaments by removing the smallest scales, which are typically in the form of hairpin vortices. Recently-proposed extensions [5] of the hairpin removal algorithm include the incorporation of renormalized Biot-Savart interaction which account for the removal (renormalization) process. 
A numerical scheme which is similar to the hairpin removal algorithm of Chorin has been recently proposed by Fernandez et al. [7]. Their simulations combine a vortex filament scheme with a 3D "filament-surgery" algorithm. The surgery is based on identifying "hairpins" (or locally-collapsed regions) with local minima of the energy density along the filament, and locally removing them by remeshing the filament.

In this paper, an alternative approach to Lagrangian large-eddy-simulation is explored. Unlike filament schemes, the present approach is based on simulating the filtered vorticity transport using a 3D particle method. Coarse computations of unstable vortex rings in three-dimensions are used to examine, qualitatively, the validity of the approach.

In addition, since vortex element schemes do not rely on structural properties of the Lagrangian mesh nor monitor changes in its topology, the computations are also used to briefly examine some computational issues concerning grid adaptivity and its role in Lagrangian LES schemes. A central question is whether an analogue to hairpin removal or filament surgery needs to be implemented in vortex element computations or whether the incorporation of dissipative subgrid scale models enables one to circumvent an apparent need for such algorithms.

\section{Formulation and numerical schemes}

As mentioned in the introduction, the present approach to Lagrangian, vorticity-based, large-eddysimulation is based on the filtered vorticity transport equation:

$$
\frac{\tilde{D} \tilde{\omega}_{i}}{D t}=\tilde{\boldsymbol{\omega}} \cdot \nabla \tilde{u}_{i}+\nu \nabla^{2} \tilde{\omega}_{i}+\frac{\partial}{\partial x_{j}}\left(\Phi_{i j}-\Phi_{j i}\right)
$$

where $\sim$ denotes spatial filtering at lengthscale $\delta, \frac{\tilde{D}}{D t} \equiv \frac{\partial}{\partial t}+\tilde{\mathbf{u}} \cdot \nabla$ is the material derivative based on the resolved velocity vector $\tilde{u}_{i}$, and $\Phi_{i j} \equiv \widetilde{\omega_{i} u_{j}}-\tilde{\omega}_{i} \tilde{u}_{j}$ is an additional vorticity (Helmholtz) stress term which accounts for the effect of unresolved velocity and vorticity fluctuations. Note that $\Phi_{i j}$ represents subgrid-scale vortex stretching and tilting due to unresolved motion while $\Phi_{j i}$ reflects vortex transport by subgrid-scale velocity fluctuations.

To complete the formulation, Eq. 1 is supplemented by the Biot-Savart law which enables us to recover the velocity field from the vorticity. For an infinite domain with no internal boundaries, this relationship takes the form:

$$
\tilde{\mathbf{u}}(\mathbf{x}, t)=-\frac{1}{4 \pi} \iiint \frac{(\mathbf{x}-\mathbf{y}) \times \tilde{\boldsymbol{\omega}}(\mathbf{y}, t)}{|\mathbf{x}-\mathbf{y}|^{3}} d^{3} \mathbf{y}
$$

where the volume integral is taken over the entire space.

Adequate implementation of Lagrangian large-eddy-simulation in the present context hinges, in particular, on: (a) a numerical scheme which is suitable for the simulation of Eqs. 1 and 2, and (b) incorporation of an appropriate subgrid scale model for the "Helmholtz stress" $\Phi_{i j}$. These two essential elements are discussed below.

\subsection{Lagrangian vortex element schemes}

Numerical simulations discussed in the following section are based on a $3 \mathrm{D}$ vortex element core solver. Construction of the scheme starts with discretization of the vorticity field into Lagrangian vortex elements of spherical overlapping cores. We use:

$$
\tilde{\boldsymbol{\omega}}(\mathbf{x}, t)=\sum_{j=1}^{N} \boldsymbol{\omega}^{j} d V^{j} f_{\delta}\left(\mathbf{x}-\chi^{j}\right)
$$


where $\boldsymbol{\omega}^{j}, d V^{j}$ and $\chi^{j}$ denote the vorticity, volume and position of the $j$ th particle, $f_{\delta}(\mathbf{x}) \equiv$ $f_{\delta}(|\mathbf{x}| / \delta) / \delta^{3}$ is the core smoothing function, and $\delta$ the core radius. The velocity field induced by the above vorticity distribution is given by the desingularized Biot-Savart law:

$$
\tilde{\mathbf{u}}(\mathbf{x}, t)=-\frac{1}{4 \pi} \sum_{j=1}^{N} \frac{\left(\mathbf{x}-\chi^{j}(t)\right) \times \boldsymbol{\omega}^{j}(t)}{\left|\mathbf{x}-\chi^{j}(t)\right|^{3}} \kappa_{\delta}\left(\mathbf{x}-\chi^{j}(t)\right)
$$

where $\kappa_{\delta}(\mathbf{x}) \equiv \kappa_{\delta}(|\mathbf{x}| / \delta)$ and $\kappa(r)=4 \pi \int_{0}^{r} x^{2} f(x) d x$ is the velocity smoothing kernel corresponding to $f$.

Evolution of the numerical solution is found by tracking the locations of the vortex elements and updating their vorticity due to local stretching, diffusion and subgrid-scale source terms, i.e. by integrating:

$$
\begin{gathered}
\frac{d \chi^{j}}{d t}=\tilde{\mathbf{u}}\left(\chi^{j}\right) \\
\frac{d \boldsymbol{\omega}^{j}}{d t}=\boldsymbol{\omega}^{j} \cdot \nabla \tilde{\mathbf{u}}\left(\chi_{i}\right)+\nu \nabla^{2} \boldsymbol{\omega}^{j}+(\nabla \cdot \Phi)^{j}
\end{gathered}
$$

In evaluating the right-hand side of Eq. 6, the velocity gradient is found by analytically differentiating Eq. 4 and evaluating the resulting expression. Following Degond and Mas-Gallic [6], the diffusion term is approximated using the Lagrangian representation of the Laplacian:

$$
\nabla^{2} \boldsymbol{\omega}^{i} \approx \frac{2}{\delta^{2}} \sum_{j=1}^{N}\left(\boldsymbol{\omega}^{j}-\boldsymbol{\omega}^{i}\right) d V^{j} g_{\delta}\left(\chi^{j}-\chi^{i}\right)
$$

where $g_{\delta}(\mathbf{x}) \equiv \delta^{-3} g(|\mathbf{x}| / \delta)$ and $g(r)=-\frac{1}{r} \frac{d f}{d r}$. In the computations, the third-order Gaussian kernel $f(r)=\frac{3}{4 \pi} \exp \left(-r^{3}\right)$ is used [1].

\subsection{Adaptive grid refinement}

The present simulations incorporate two local mesh "refinement" schemes. The first is essentially an element-splitting algorithm which aims at ensuring that neighboring elements maintain overlapping cores at all times. Its construction mimics filament-based algorithms discussed in $[9,10]$. However, it differs from these previous constructions in that the local mesh refinement operations, and criteria for when to perform them, are independent of the topological features of the Lagrangian grid. This feature is desirable since, in general, 3D particle methods need not rely on book-keeping of how vortex elements are inter-connected.

The element splitting algorithm is based on associating with each vortex element, $\chi^{j}$, three vectors, $\delta \chi^{j, k}, k=1,2,3$. At the start of the computations, the three vectors are mutually orthogonal. Their evolution is found by integrating the equations of motion:

$$
\frac{d \delta \chi^{j, k}}{d t}=\delta \chi^{j, k} \cdot \nabla \mathbf{u}\left(\chi^{j}\right)
$$

The straining of the vectors by the strain field is monitored during the computations. When the length of any of the three vectors exceeds a predetermined value, $h_{\max } \equiv \alpha \delta$, the element is split into two in that vector's direction. To describe the local remeshing procedure, we assume without loss of generality that $\left|\delta \chi^{j, 1}\right|>h_{\max }$; then the splitting algorithm: (1) splits the $j$ th element into 
two elements located at $\chi^{j} \pm \delta \chi^{j, 1} / 2$, each having half the strength of the original element, and (2) associates with each of the new elements the three vectors, $\delta \chi^{j, 1} / 2, \delta \chi^{j, 2}$ and $\delta \chi^{j, 3}$.

One of the "side effects" of the splitting algorithm is that, when the flow experiences severe stretching, it may lead to rapid and excessive growth in the number of elements. To control this phenomenon, and maintain the number of degrees of freedom at a feasible level, an element recombination scheme is also incorporated. Briefly, this algorithm monitors the distance between neighboring elements and consolidates two elements into one whenever their separation distance falls below $h_{\min } \equiv \beta \delta$, where $\beta<1$ is also a predetermined factor. The strength of the consolidated element is the sum of the strengths of its "parents", i.e. $\boldsymbol{\omega} d V=\boldsymbol{\omega}^{1} d V^{1}+\boldsymbol{\omega}^{2} d V^{2}$. The consolidated element is located at the centroid of the original particles weighted using the strength of parents, $\chi=$ $\frac{\chi^{1}\left|\boldsymbol{\omega}^{1} d V^{1}\right|+\boldsymbol{\chi}^{2}\left|\boldsymbol{\omega}^{2} d V^{2}\right|}{\left|\boldsymbol{\omega}^{1} d V^{1}\right|+\left|\boldsymbol{\omega}^{2} d V^{2}\right|}$. Construction of the algorithm is completed by specifying the three "length" vectors associated with the "recombined" element. As schematically illustrated in Fig. 1, these are determined by first fitting an ellipsoid around the original elements and then identifying the three vectors with the axes of the ellipsoid. Thus, the splitting and recombination algorithms are fully compatible with each other.

Finally, we note that both the splitting and recombination schemes leave the total volume of vorticity unchanged. However, neither the kinetic energy nor the enstrophy is explicitly preserved during these operations.

\subsection{Sub-filter scale models}

So far, we have primarily experimented with Smagorinsky-type sub-filter scale models. The corresponding vorticity source term is expressed in terms of an isotropic, but non-uniform, eddy diffusivity, $\nu_{T}$. By analogy with the momentum equation, the Helmholtz stress is related to the gradient of the resolved vorticity through:

$$
\Phi_{i j}=\nu_{T} \frac{\partial \tilde{\omega}_{i}}{\partial x_{j}}
$$

while the eddy diffusivity $\nu_{T}$ is expressed as:

$$
\nu_{T}=\left(c_{T} \delta\right)^{2} \sqrt{2 \tilde{S}_{i j} \tilde{S}_{i j}}
$$

where $\tilde{S}_{i j}$ is the resolved strain-rate tensor and $c_{T}$ is a constant. Note that the filter size $\delta$ has been identified with the core size of the vortex elements.

In the form summarized above, implementation of the Smagorinsky model requires an input value for $c_{T}$. In order to obtain a reasonable estimate, we consider the equilibrium conditions for a homogeneous, isotropic turbulence field. At high Reynolds number, the filtered enstrophy equation reduces to a balance between enstrophy production by the large-scale motion and enstrophy dissipation by sub-filter scale Helmholtz stresses; assuming homogeneity and stationary flow, we have:

$$
\left\langle\tilde{\omega}_{i} \tilde{\omega}_{j} \tilde{S}_{i j}\right\rangle \approx\left\langle\nu_{T} \frac{\partial \tilde{\omega}_{i}}{\partial x_{j}} \frac{\partial \tilde{\omega}_{i}}{\partial x_{j}}\right\rangle
$$

Substituting Eq. 10 for the sub-filter scale Helmholtz stress leads to:

$$
\left\langle\tilde{\omega}_{i} \tilde{\omega}_{j} \tilde{S}_{i j}\right\rangle \approx\left(c_{T} \delta\right)^{2}\left\langle\sqrt{2 \tilde{S}_{m n} \tilde{S}_{m n}} \frac{\partial \tilde{\omega}_{i}}{\partial x_{j}} \frac{\partial \tilde{\omega}_{i}}{\partial x_{j}}\right\rangle
$$


Next, assuming that the strain rate norm and the vorticity gradient norm are uncorrelated, and that $\left\langle\sqrt{\tilde{S}_{m n} \tilde{S}_{m n}}\right\rangle \approx\left\langle\tilde{S}_{m n} \tilde{S}_{m n}\right\rangle^{1 / 2}$, we get:

$$
\left\langle\sqrt{2 \tilde{S}_{m n} \tilde{S}_{m n}} \frac{\partial \tilde{\omega}_{i}}{\partial x_{j}} \frac{\partial \tilde{\omega}_{i}}{\partial x_{j}}\right\rangle \approx \sqrt{2}\left\langle\tilde{S}_{m n} \tilde{S}_{m n}\right\rangle^{1 / 2}\left\langle\frac{\partial \tilde{\omega}_{i}}{\partial x_{j}} \frac{\partial \tilde{\omega}_{i}}{\partial x_{j}}\right\rangle
$$

A similar assumption was made by Lilly [14] and Scotti et al. [17] and has been shown to be a good approximation Cerutti et al. [2] from analysis of DNS of isotropic turbulence. Kinematic relations for isotropic, incompressible turbulence give us:

$$
\begin{gathered}
\left\langle\tilde{\omega}_{i} \tilde{\omega}_{j} \tilde{S}_{i j}\right\rangle=-\frac{35}{2}\left\langle\left(\frac{\partial \tilde{u}_{1}}{\partial x_{1}}\right)^{3}\right\rangle \\
\left\langle\tilde{S}_{i j} \tilde{S}_{i j}\right\rangle=-\frac{15}{2}\left\langle\left(\frac{\partial \tilde{u}_{1}}{\partial x_{1}}\right)^{2}\right\rangle
\end{gathered}
$$

Substituting Eq. 15 into Eq. 13, and Eqs. 13 and 14 into Eq. 12 produces the equation

$$
-\frac{35}{2}\left\langle\left(\frac{\partial \tilde{u}_{1}}{\partial x_{1}}\right)^{3}\right\rangle \approx\left(c_{T} \delta\right)^{2} \sqrt{15}\left\langle\left(\frac{\partial \tilde{u}_{1}}{\partial x_{1}}\right)^{2}\right\rangle^{1 / 2}\left\langle\frac{\partial \tilde{\omega}_{i}}{\partial x_{j}} \frac{\partial \tilde{\omega}_{i}}{\partial x_{j}}\right\rangle
$$

It follows that

$$
c_{T}^{2} \approx \frac{35}{15^{3 / 2}}\left|S_{3}\right| \frac{\left\langle\tilde{S}_{i j} \tilde{S}_{i j}\right\rangle}{\delta^{2}\left\langle\frac{\partial \tilde{\omega}_{m}}{\partial x_{n}} \frac{\partial \tilde{\omega}_{m}}{\partial x_{n}}\right\rangle}
$$

where $S_{3}=\left\langle\left(\frac{\partial \tilde{u}_{1}}{\partial x_{1}}\right)^{3}\right\rangle /\left\langle\left(\frac{\partial \tilde{u}_{1}}{\partial x_{1}}\right)^{2}\right\rangle^{3 / 2}$ is the skewness of the filtered velocity derivative. The energy spectra of homogeneous, isotropic turbulence provide the ratio of the norms of the filtered strain rate and filtered vorticity gradient:

$$
\frac{\left\langle\tilde{S}_{i j} \tilde{S}_{i j}\right\rangle}{\delta^{2}\left\langle\frac{\partial \tilde{\omega}_{m}}{\partial x_{n}} \frac{\partial \tilde{\omega}_{m}}{\partial x_{n}}\right\rangle}=\frac{\int_{0}^{\infty} 2|F(k)|^{2} k^{2} E(k) d k}{\delta^{2} \int_{0}^{\infty} 2|F(k)|^{2} k^{4} E(k) d k}
$$

where $E(k)$ is the turbulence 3D energy spectrum and $|F(k)|^{2}$ is the filter spectrum. Since it is assumed that the filter cuts off somewhere within the inertial range, we may use the Kolmogorov spectrum $E(k)=C_{k} \epsilon^{2 / 3} k^{-5 / 3}$ with $C_{k}=1.6$. The filter $F(k)$ is taken to be the Fourier transform of the 3rd order Gaussian function used in the particle discretization. Numerical integration gives $\left\langle\tilde{S}_{i j} \tilde{S}_{i j}\right\rangle / \delta^{2}\left\langle\frac{\partial \tilde{\omega}_{m}}{\partial x_{n}} \frac{\partial \tilde{\omega}_{m}}{\partial x_{n}}\right\rangle \doteq 0.1124$. The skewness $S_{3}$ can be measured by filtering experimental or DNS velocity signals. While a dependence on filter size was observed in Cerrutti et al. [2], a representative value is $S_{3} \approx-0.4$. Using $\left|S_{3}\right|=0.4$ leads to $c_{T}=0.15$, in reasonable agreement with the standard Smagorinsky model.

\section{Results and discussion}

The construction summarized above followed tests of several other approaches. We first highlight the essential elements of this exercise and then present results for the models of Section 2. 
All applications discussed in the present study focus on the three-dimensional motion of an isolated vortex ring in the limit of high Reynolds number. Selection of this physical setting is motivated by the fact that the vortex ring is unstable to azimuthal perturbations [19] whose rapid growth leads to the formation of a complex "turbulent" vortical structure (see e.g. Fig. 6.56 in [15]). Thus, the setting proves ideal for our present purpose, since one of the primary objectives of Lagrangian LES is to be able to model the formation of complex vortical structures and to capture their large-scale features while using very coarse computational grids. Unfortunately, one of the disadvantages of the setting is that smallscale turbulence and the behavior of Helmholtz stresses are hard to characterize. This is the case because (1) the isotropy and/or homogeneity assumptions which form the basis of most analytical or semi-empirical predictions do not apply, and (2) experimental measurements of the 3D vector fields are scarce and interpretation of the data is difficult, in part due to the extreme sensitivity of the flow to perturbations and initial conditions. Briefly, we have sacrificed detailed quantitative analysis of the performance of subgridscale turbulence models in order to examine the behavior of the scheme in a complex, challenging environment.

\subsection{Summary of preliminary tests}

Preliminary computations have focused on two essential issues: (1) the need for adaptive mesh refinement, and (2) the (qualitative) role of subgrid modeling in the context of a coarse Lagrangian computation. Highlights of this exercise are summarized as follows:

1. In all cases considered, we find it necessary to implement a mesh refinement scheme which locally redistributes elements in regions of high strain. Without the redistribution scheme, the accuracy of the computations deteriorates as elements are depleted from regions of high strain (e.g. $[9,10])$; this phenomenon eventually leads to breakdown of the computations.

While similar experiences have been reported in a number of previous studies, we wished to establish whether incorporation of a subgrid scale model could eliminate the need for local mesh refinement. Specifically, we have attempted to apply non-adaptive large-eddy simulations using a constant eddy-diffusivity Smagorinsky model. However, even when artificially large model constants were used, the computations exhibited the same loss-of-resolution symptoms which were observed in inviscid non-adaptive calculations. Thus, incorporation of a mesh refinement scheme to cure these symptoms is needed for the present conditions, whether or not a subgrid scale model is used.

2. The local refinement scheme used in the present study is based on splitting of the vortex elements. As shown in Fig. 2, this simple splitting algorithm leads to a rapid rise in the number of vortex elements. Incorporation of subgrid-scale turbulence model slows down the increase in the number of elements, but does not alter the explosive-type growth which is observed soon after the start of the calculations.

3. Following the above remarks, it became obvious to us that, in order to successfully implement Lagrangian large eddy simulation of a complex flow while maintaining the number of elements at a reasonable level, an element removal scheme was also necessary. We have considered two such schemes: the first is a simple remeshing algorithm which is discussed below. The second is the recombination algorithm summarized in Section 2.

4. The remeshing scheme which we constructed aims at eliminating "unnecessary" degrees of freedom by interpolating the numerical solution onto a Eulerian grid. The grid points belong to a regular square mesh and cover the region where the local magnitude of vorticity exceeds $1 \%$ of the peak vorticity value, and the interpolation is performed such that the total volume of vorticity and the total enstrophy are conserved.

While the remeshing step was found to significantly reduce the number of elements, explosive 
growth in the number of elements still prevailed between successive remeshing steps. In addition, the diffusive nature of the Eulerian remeshing scheme led to an artificial growth in the vorticity region. The effect was quite damaging in coarse simulations such as those targeted here, and the present simple splitting/remeshing scheme was not able to maintain a reasonably small number of elements.

We have not attempted to enhance the remeshing scheme by introducing an "anti-diffusion" algorithm (e.g. $[8,18]$ ) which may prevent the artificial growth in the vorticity region. Rather, we have pursued development of the Lagrangian recombination scheme described in Section 2.

5. When the splitting and recombination algorithms are incorporated, the resulting model bears strong resemblance to Chorin's hairpin-removal algorithm [3], and to the filament surgery scheme of Fernandez et al. [7]. However, the present vortex element scheme differs from filament-based algorithms in several important aspects. Briefly, the vortex element scheme is less efficient than filament-based computations since, in addition to element velocities, the velocity gradient and the strain-dependent diffusion terms must also be evaluated. On the other hand, the scheme extends more easily to more general flow conditions, including finite Reynolds number, stratified and/or reacting flow $[11,12]$.

\subsection{Sample results}

Details are now provided for some computations performed using the models described in Section 2. As mentioned earlier, an unbounded vortex ring is selected. Initially, the ring is axisymmetric and the core vorticity distribution is assumed to be given by:

$$
\Omega(\rho)=\frac{1}{a \sigma^{2}} \exp \left(-\frac{\rho^{3}}{\sigma^{3}}\right)
$$

where $\Omega$ is the azimuthal vorticity, $\rho$ is the distance in an azimuthal plane measured from the center of the vortex core, $\sigma$ is the standard deviation of the Gaussian profile. We choose $a=\pi \gamma(2 / 3) / 3$ so that the vortex ring has normalized circulation $\Gamma=2$, and restrict our attention to a "fat" vortex ring with core to radius ratio, $\sigma / R=0.45$.

At the start of the computations, the radius of the vortex ring is perturbed in the azimuthal direction using sinewaves of small amplitude. We use:

$$
R=R_{0}\left[1+\epsilon_{1} \sin \theta+\epsilon_{n} \sin (n \theta)\right]
$$

where $R_{0}$ is the undisturbed ring radius. Thus, a two-mode azimuthal perturbation is used. The wavenumber $n$ is chosen to coincide with the wavenumber of the most unstable azimuthal bending mode [19]. For the presently-selected vorticity profile and core to radius ratio, $n=7$ [9]. Meanwhile, the bending mode with unity wavenumber is used to break the symmetry of the unstable mode. Relative perturbation amplitudes $\epsilon_{n}=2 \%$ and $\epsilon_{1}=0.4 \%$ are used.

Discretization of the perturbed vorticity distribution is performed using a uniform grid of mesh size $h / R_{0}=0.1375$. Grid points are used in the region where the vorticity magnitude exceeds $1 \%$ of the corresponding peak value. Initial vortex strengths are calculated by averaging the vorticity distribution within each grid cell. The core radius of the vortex elements $\delta / R_{0}=0.175$ and the Lagrangian field initially consists of 4310 particles. The parameters for element splitting and recombination are $\alpha=1$ and $\beta=0.25$.

Figure 3 illustrates the evolution of the vortex ring, as predicted using a Smagorinsky Helmholtz stress model with $c_{T}=0.15$. The figure reflects the growth of unstable waves which non-uniformly deform the core of the vortex ring. It also shows the formation of a complex wake structure behind 
the concentrated eddy core, and that this wake consists of hairpin-like structures which extend in the streamwise direction. The qualitative resemblance between the present predictions and experimental visualizations of turbulent vortex ring (e.g. [15]) is very encouraging.

The performance of the splitting/recombination algorithm is also quite encouraging. As shown in Fig. 4, when the recombination algorithm is incorporated, the growth in the number of elements changes from an explosive-type growth to a substantially-slower, controlled rise. Comparison of Figs. 3 and 4 indicates that the rise in the number of elements is proportionate to the extension of wake behind the vortex ring. In fact, Fig. 3 suggests that the number of elements may be further reduced, since the large number of elements present in the wake of the vortex ring carry only a small fraction of the total vorticity. Presumably, elimination of elements carrying minute values of vorticity can be performed in order to speed up the computations with minimal impact on the accuracy of the predictions. We have not, however, attempted to implement such an element removal procedure.

Additional insight into the impact of the splitting/recombination algorithm is gained by comparing the above model predictions with those obtained without subgrid scale model $\left(c_{T}=0\right)$. Figures 5 and 6 show the evolution of total enstrophy and kinetic energy, respectively; predictions for both $c_{T}=0$ and $c_{T}=0.15$ are plotted. The figures indicate that the splitting/recombination algorithm is in itself diffusive since both enstrophy and kinetic energy decay during the later stages of the computations, even when the subgrid model is not employed. However, the splitting/recombination algorithm does not account for all the effective diffusion of the computational model, since differences exist between curves for $c_{T}=0$ and $c_{T}=0.15$. In particular, the decay rates of enstrophy and kinetic energy at the late stages are larger when a subgrid scale model is used.

The impact of the eddy diffusivity model is better illustrated by contrasting computed results on the structure of the vortex ring at the later stages. Figure 7 compares vorticity contours in azimuthal and streamwise planes obtained using $c_{T}=0$ and $c_{T}=0.15$. It clearly shows that both the subgrid model and the splitting/recombination algorithm have an appreciable impact on the computations.

\section{Closing remarks}

This paper discusses initial efforts aiming at the construction of Lagrangian LES schemes. The approach adopted here is based on the filtered vorticity transport equation and the representation of Helmholtz stresses in terms of a Smagorinsky eddy diffusivity scheme. Computations of unstable vortex rings in three-dimensions show that the model can capture the large-scale features of the flow using a relatively small number of elements. Thus, the present effort is quite encouraging.

During the course of the present study, implementation of algorithms which maintain adequate and efficient discretization by judiciously introducing and eliminating elements has emerged as a highly-desirable feature, at least for simulations on coarse grids. This experience is especially relevant to large-eddy models since these models under-resolve, by design, the smallest lengthscales.

While computational techniques which are suitable for large eddy simulation are developing rapidly, the modeling of subgrid scale Helmhotlz stresses in a Lagrangian setting is still in its infancy. Not surprisingly, several crucial questions still remain, concerning the need for subgrid models, their impact on the predictions, and their relationship to grid refinement schemes. These questions will be addressed in future work. 


\section{References}

[1] Beale, J.T. and Majda, A., "High Order Accurate Vortex Methods with Explicit Velocity Kernels," J. Comp. Phys., 58, pp. 188, 1985.

[2] Cerutti, S., Meneveau, C. and Knio, O.M., Manuscript in preparation (1996).

[3] Chorin, A.J., "Hairpin Removal in Vortex Interactions II," J. Comp. Phys., 107, pp. 1, 1993.

[4] Chorin, A.J., Vorticity and Turbulence, Springer, New York, 1993.

[5] Chorin, A.J., "Microstructure, Renormalization, and More Efficient Vortex Methods", Vortex Flows and Related Numerical Methods II, edited by Y. Gagnon et al., ESAIM: Proc., 1, http://www.emath.fr/proc/Vol.1/, pp. 1-14, 1996.

[6] Degond, P. and Mas-Gallic, S., "The Weighted Particle Method for Convection-Diffusion Equations. Part 1: The Case of an Isotropic Viscosity," Math. Comp., 53, pp. 485, 1989.

[7] Fernandez, V.M., Wu, L., Zabusky, N.J., Liu, P., Bhatt, S. and Gerasoulis, A., "Filament Surgery and Temporal Grid Adaptivity Extensions to a Parallel Tree Code for Simulation and Diagnosis in 3D Vortex Dynamics," Proc. Sec. Int'l. Workshop on Vortex Flows and Related Numerical Methods, Montréal, Canada, pp. 90-91, 1995.

[8] Huberson, S., Jolles, A. and Shen, W.-Z., "Numerical Simulation of Incompressible Viscous Flows by Means of Particle Methods," Lec. Appl. Math., 28, pp. 369, 1991.

[9] Knio, O.M. and Ghoniem, A.F., "Numerical Study of a Three-Dimensional Vortex Method," J. Comp. Phys., 86, pp. 75, 1990.

[10] Knio, O.M. and Ghoniem, A.F., "Three-Dimensional Vortex Simulation of Rollup and Entrainment in a Shear Layer," J. Comp. Phys., 97, pp. 172, 1991.

[11] Knio, O.M. and Ghoniem, A.F., "The Three-Dimensional Structure of Periodic Vorticity Layers Under Non-Symmetric Conditions," J. Fluid Mech., 243, pp. 353, 1992.

[12] Knio, O.M. and Ghoniem, A.F., "Vortex Simulation of a Three-Dimensional Reacting Shear Layer with Infinite-Rate Kinetics," AIAA J., 30, pp. 105, 1992.

[13] Leonard, A., "Energy Cascade in Large-Eddy Simulations of Turbulent Fluid Flows," Adv. Geophys., 18, pp. 237, 1974.

[14] Lilly, D.K., "The Representation of Small-Scale Turbulence in Numerical Simulation Experiments," Proc. IBM Scientific Computing Symposium on Environmental Sciences, p. 195, 1967.

[15] Lugt, H.J., Vortex Flow in Nature and Technology, Wiley, New York, p. 123, 1983.

[16] Rogallo, R. and Moin, P., "Numerical Simulation of Turbulent Flows," Ann. Rev. Fluid. Mech., 16, pp. 99, 1984.

[17] Scotti, A., Meneveau, C. and Lilly, D.K., "Generalized Smagorinsky Model for Anisotropic Grids," Phys. Fluids A, 5, pp. 2306, 1993.

[18] Soteriou, M.C. and Ghoniem, A.F., "Numerical Simulation of Exothermic Spatially Developing Shear Flows using the Lagrangian Transport-Element Method," Proc. Sec. Int'l. Workshop on Vortex Flows and Related Numerical Methods, Montréal, Canada, p. 57, 1995. 
[19] Widnall, S.E. and Tsai, C.-Y., "The Instability of the Thin Vortex Ring of Constant Vorticity," Proc. Roy. Soc. London A1334, 287, pp. 273, 1977.

\section{parent elements}

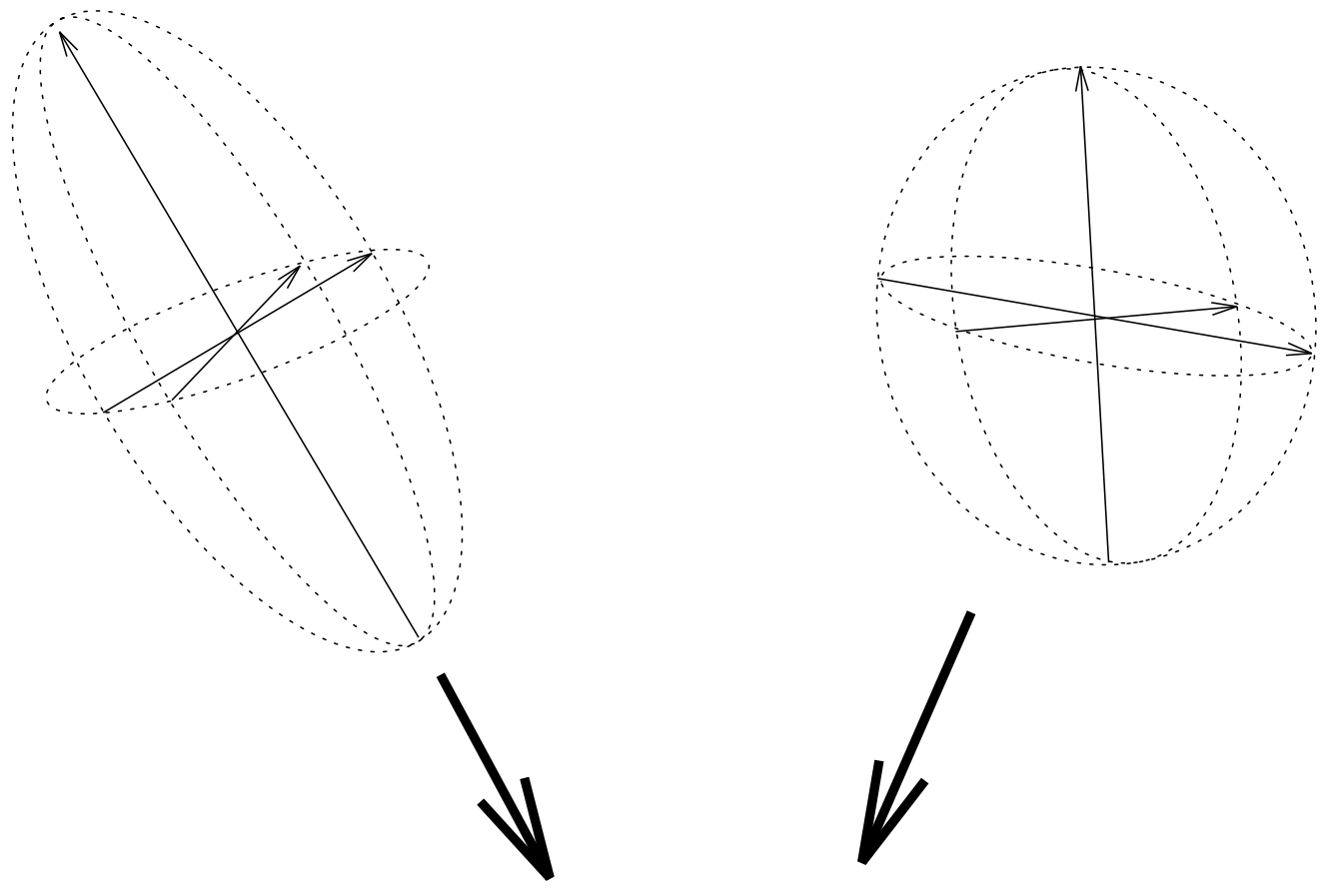

consolidated element

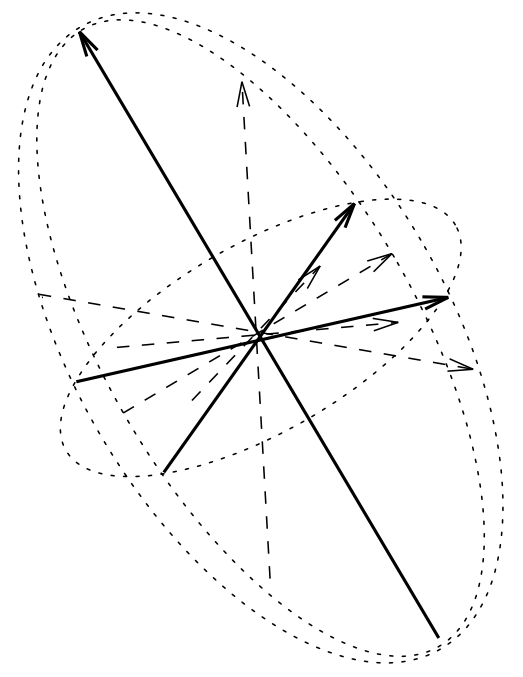

Figure 1: Schematic illustration of the action of the recombination algorithm. 


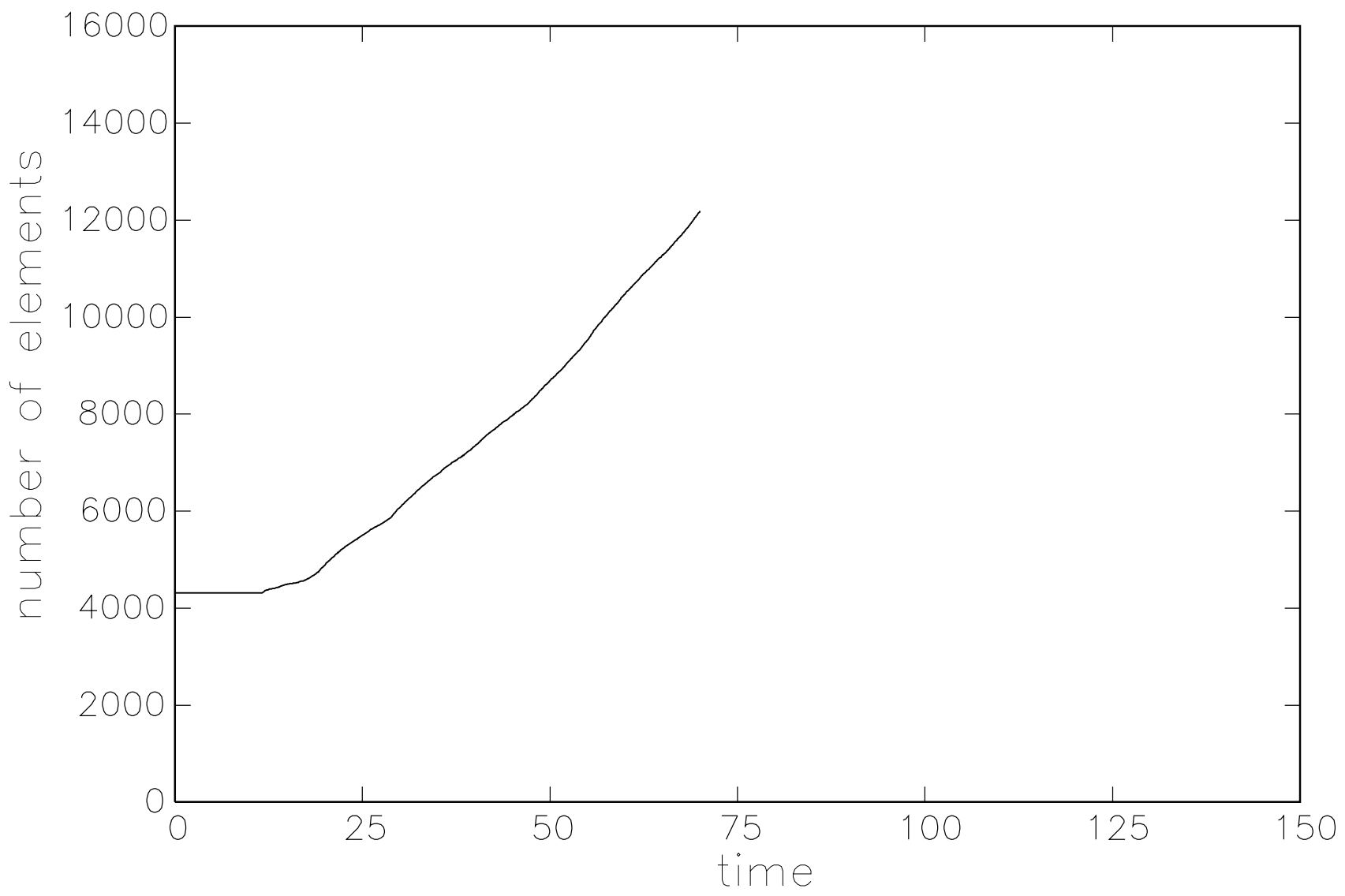

Figure 2: Number of vortex elements in an adaptive 3D simulation of an unstable vortex ring. 

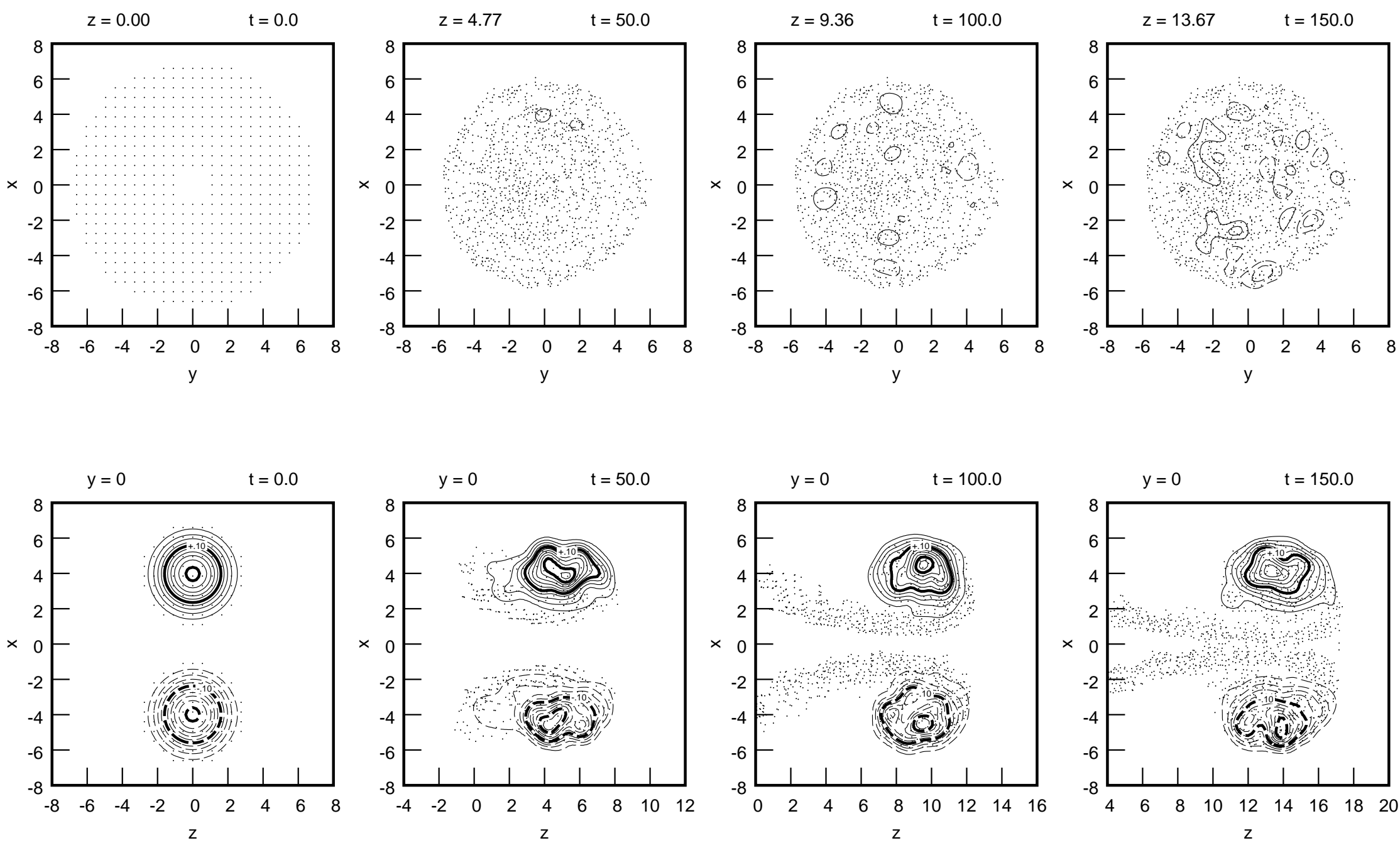

Figure 3: Contours of streamwise (top) and azimuthal (bottom) vorticity in the core of the vortex ring. Computations are performed using a Lagrangian LES scheme with Smagorinsky eddy viscosity. The times at which the frames are generated are indicated. 


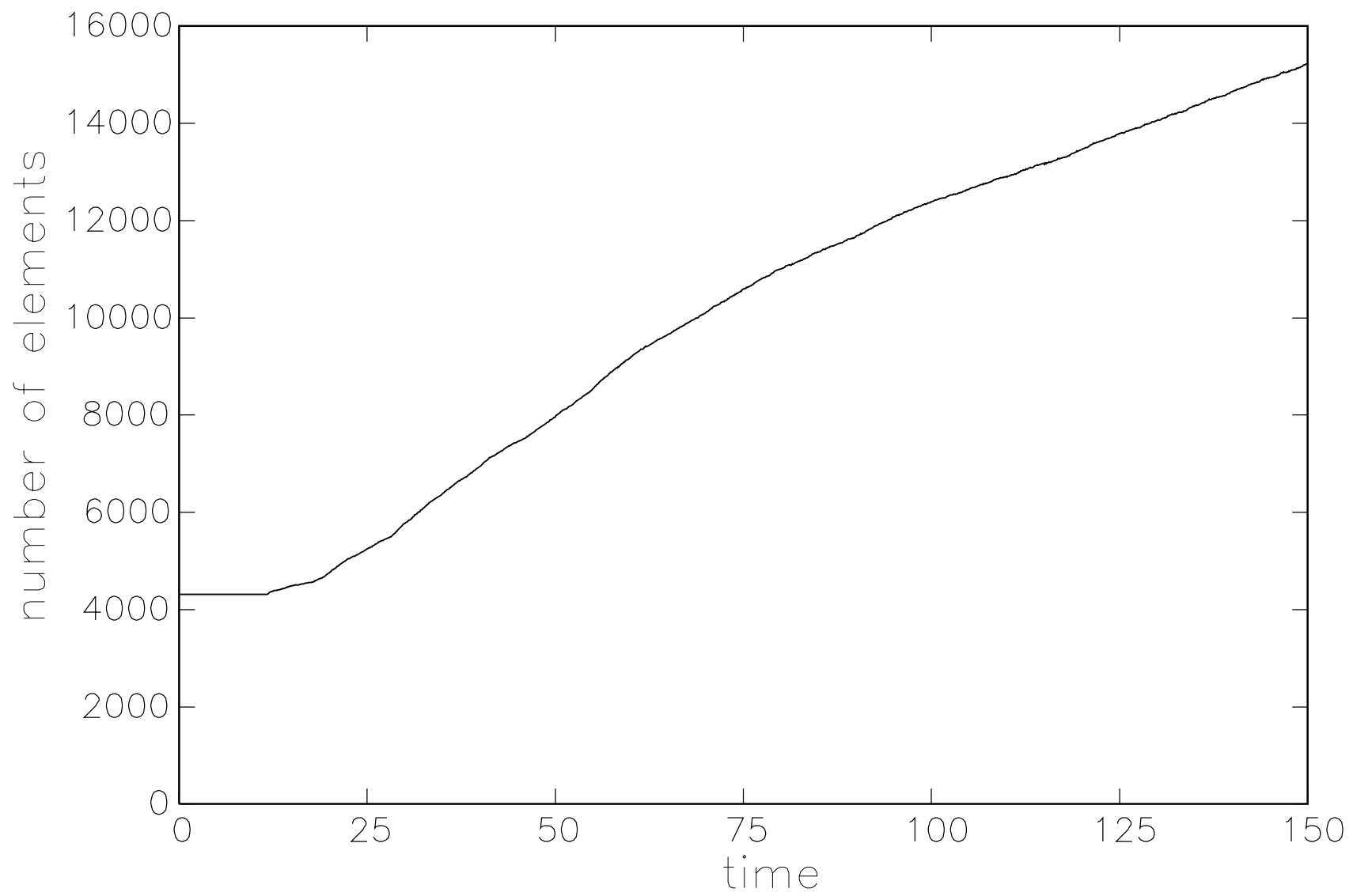

Figure 4: Number of elements in a simulation with splitting and recombination. 


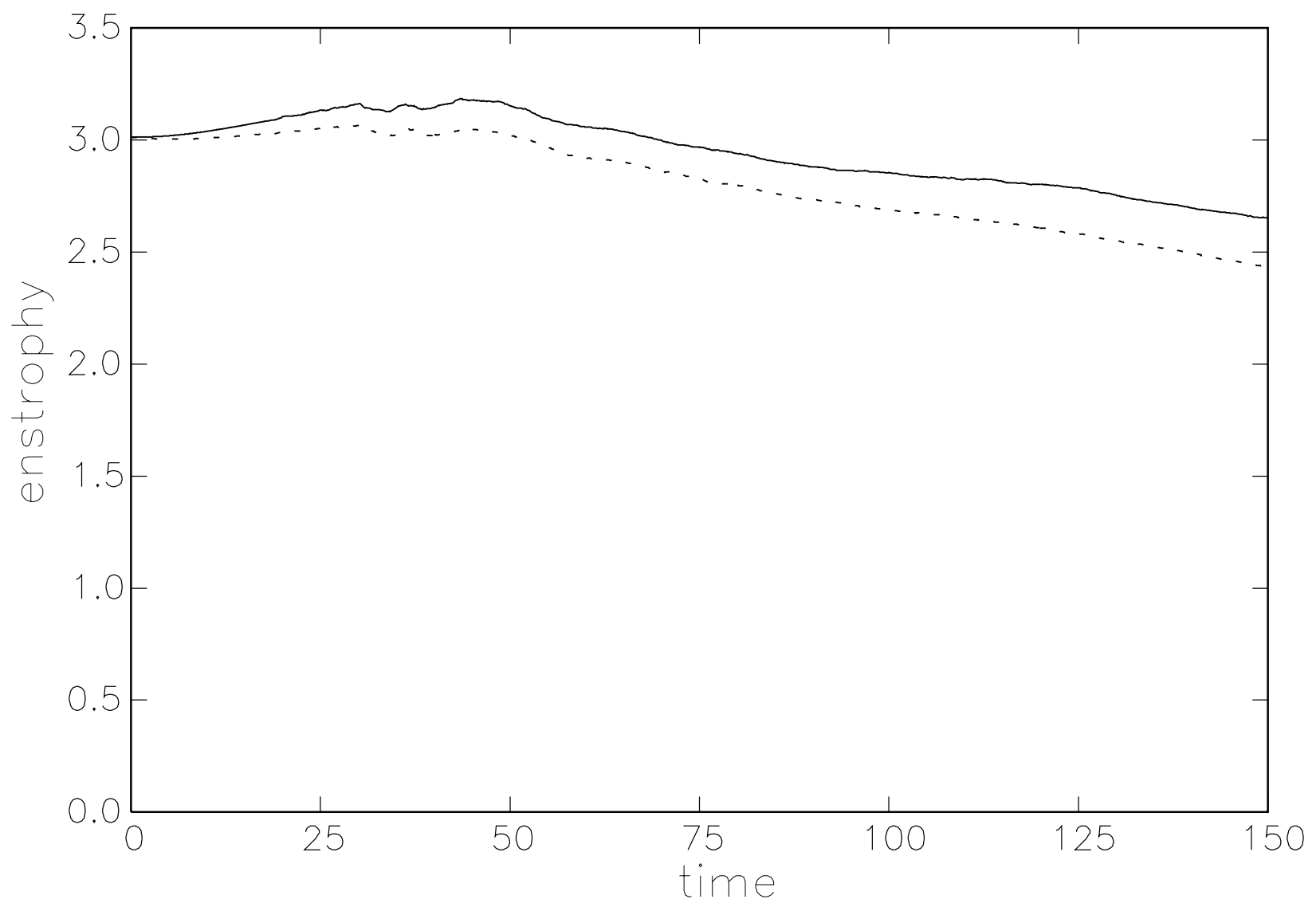

Figure 5: Evolution of the enstrophy in Lagrangian LES schemes: using a Smagorinsky eddydiffusivity model (dash), without subgrid scale model (solid). 


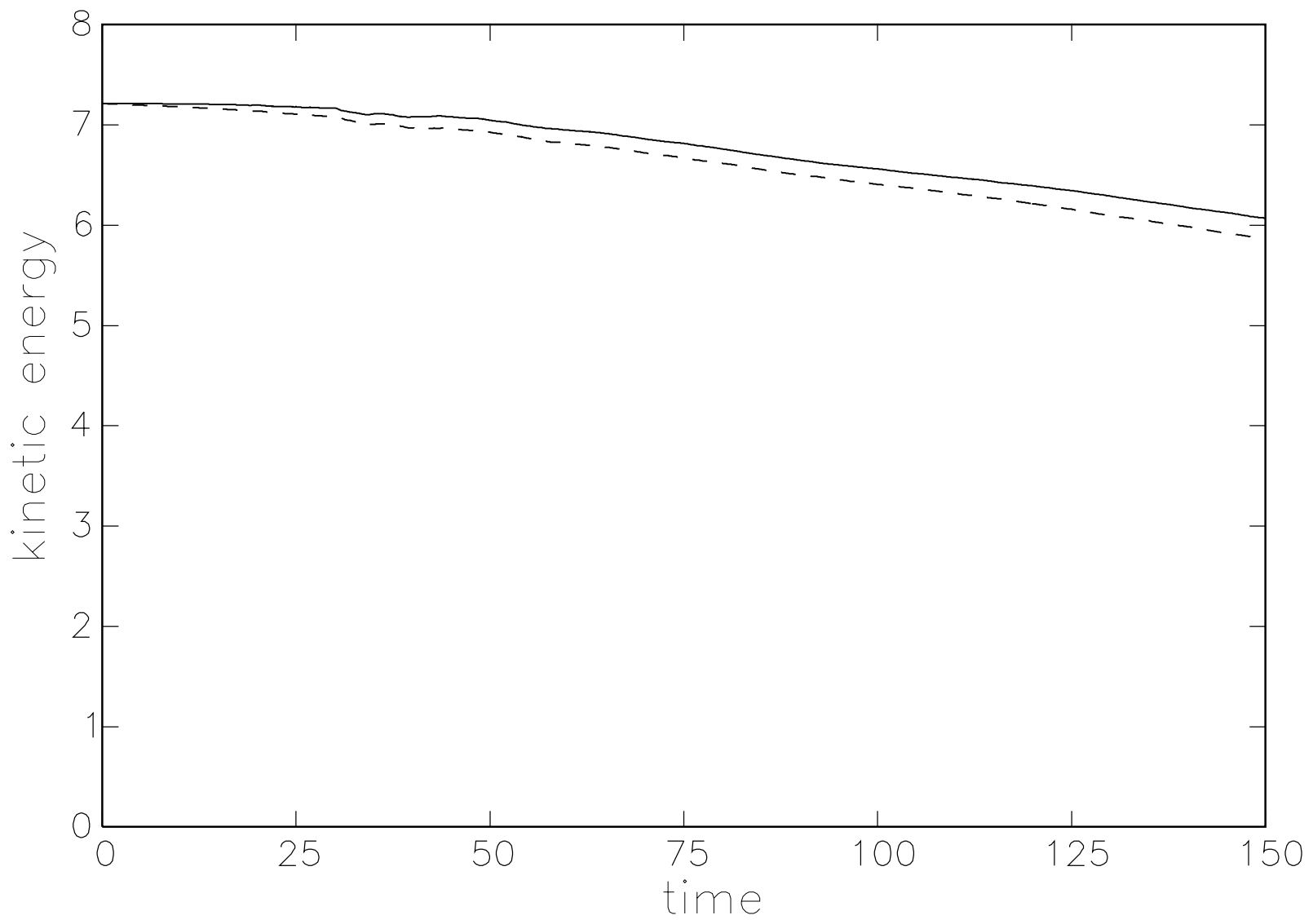

Figure 6: Evolution of the total kinetic energy in Lagrangian LES schemes: using a Smagorinsky eddy-diffusivity model (dash), without subgrid scale model (solid). 

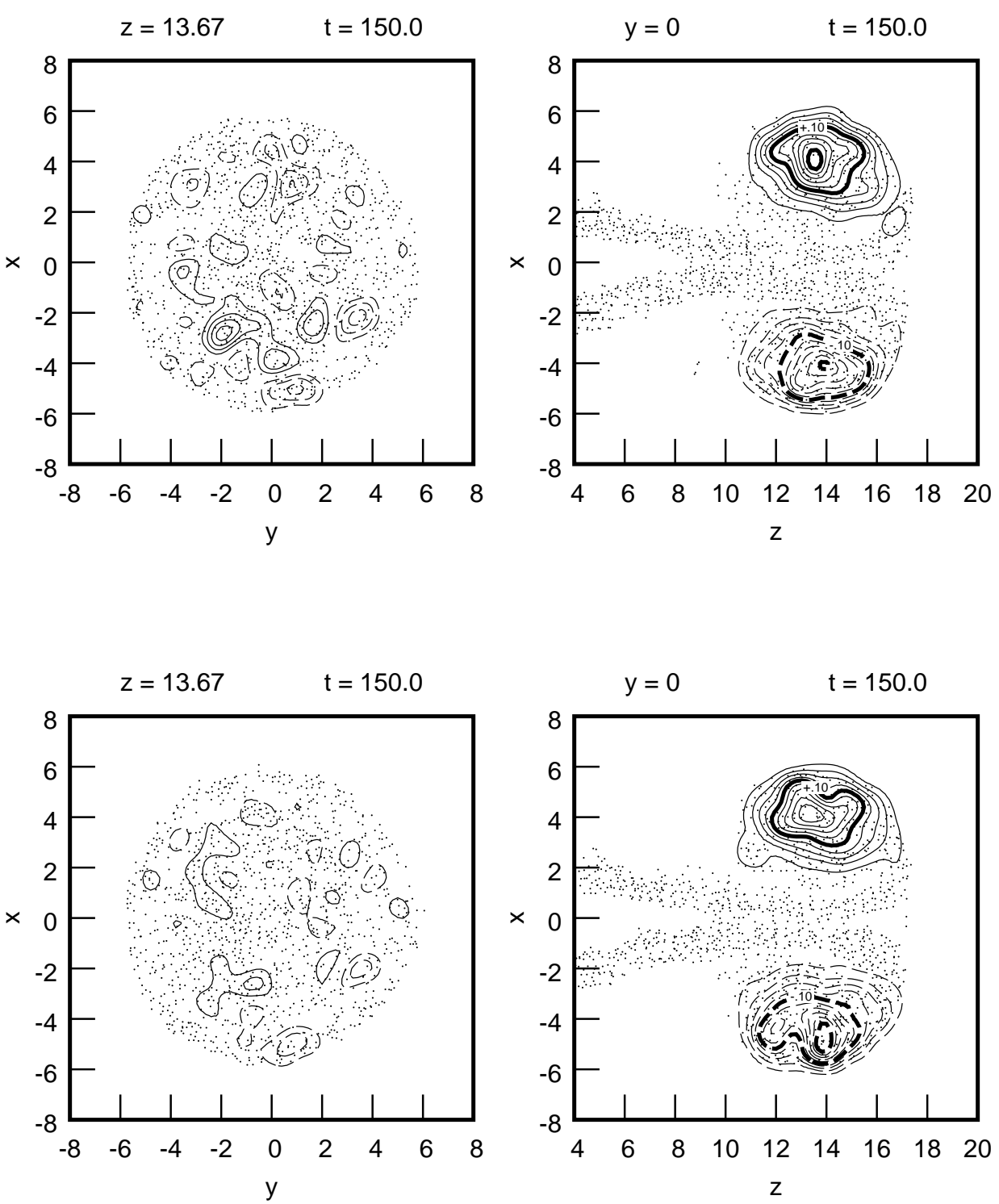

Figure 7: Contours of azimuthal (left) and streamwise (right) vorticity at $t=150$ in Lagrangian LES computations. The top row corresponds to a simulation which uses a Smagorinsky model, while the bottom row is obtained using a simulation with no subgrid model. 